\title{
Psychological aspects of the menopause
} Anke Rohde

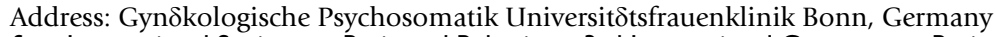 \\ from International Society on Brain and Behaviour: 3rd International Congress on Brain and Behaviour \\ Thessaloniki, Greece. 28 November - 2 December 2007 \\ Published: 17 April 2008 \\ Annals of General Psychiatry 2008, 7(Suppl I):S72 doi:10.1186/1744-859X-7-SI-S72
}

This abstract is available from: http://www.annals-general-psychiatry.com/content/7/SI/S72

(c) 2008 Rohde; licensee BioMed Central Ltd.

A range of symptoms may impact women during the menopausal transition - several psychological symptoms (depressive mood, irritability, affective lability, lack of concentration) but also vasomotor symptoms like hot flushes, palpitations and sweating or somatic symptoms (sleep disorders, vaginal dryness often leading to pain during sexual intercourse, loss of energy, and joint and muscle pain). Loss of libido and other sexual problems may result and may also lead to depression. The impact of perimenopausal symptoms on the feeling of well-being and the quality of life of women depends not only on the frequency and severity of symptoms, but also on the individual attitude towards loss of fertility and aging. Cultural beliefs and the ability to cope with oncoming somatic and social changes are also important.

In terms of psychological symptoms, there seems to be an individual vulnerability: women with a history of mental problems are more likely to develop perimenopausal psychiatric symptoms. However, even prior mentally healthy women seem to have an increased risk of developing perimenopausal mental problems, as recent studies have shown. From small studies and especially from clinical experience, we know that hormonal replacement treatment (HRT) is able to reduce mild depression and other psychological symptoms. With the changing awareness of women regarding the dangers of HRT there may be an increasing number of women who seek alternative treatment. If antidepressant medication is considered, an agent should be chosen which also gives the chance to influence vasomotor and somatic symptoms. Because perimenopausal symptoms are typically multifactorial in nature, it is important to consider (as well as the biological mechanism and hormonal influences) coping strategies, individual living situation, and self-perception regarding health. The special needs of the individual woman, therefore, need to be considered in order that treatment decisions are made in the sense of shared decision making. 